\title{
Omega-3 polyunsaturated fatty acids in the prevention of postoperative complications in colorectal cancer: a meta-analysis
}

This article was published in the following Dove Press journal:

OncoTargets and Therapy

9 December 2016

Number of times this article has been viewed

Hai Xie'

Yan-na Chang²

'Department of Emergency, The First Hospital of Lanzhou University, 2Department of Anesthesiology, Affiliated Hospital of Gansu University of Chinese Medicine, Lanzhou, People's Republic of China

Correspondence: Yan-na Chang Department of Anesthesiology, Affiliated Hospital of Gansu University of Chinese Medicine, 732 West Jiayuguan Road, Chengguan, Lanzhou, Gansu 730000, People's Republic of China

Tel +86931 8635008

Email xiehai_md@tom.com
Objective: To evaluate systematically the clinical efficacy of omega-3 polyunsaturated fatty acids (PUFAs) in the prevention of postoperative complications in colorectal cancer (CRC) patients.

Materials and methods: Published articles were identified by using search terms in online databases - PubMed, Embase, and the Cochrane Library - up to March 2016. Only randomized controlled trials investigating the efficacy of omega-3 PUFAs in CRC were selected and analyzed through a meta-analysis. Subgroup, sensitivity, and inverted funnel-plot analyses were also conducted.

Results: Eleven articles with 694 CRC patients were finally included. Compared with control, omega-3 PUFA-enriched enteral or parenteral nutrition during the perioperative period reduced infectious complications (risk ratio [RR] 0.63, 95\% confidence interval [CI] 0.47-0.86; $P=0.004$ ), tumor necrosis factor alpha (standard mean difference [SMD] $-0.37,95 \% \mathrm{CI}-0.66$ to -0.07 ; $P=0.01$ ), interleukin-6 (SMD $-0.36,95 \% \mathrm{CI}-0.66$ to $-0.07 ; P=0.02$ ), and hospital stay (MD $-2.09,95 \% \mathrm{CI}-3.71$ to $-0.48 ; P=0.01)$. No significant difference was found in total complications, surgical site infection, or $\mathrm{CD} 4^{+}: \mathrm{CD} 8^{+}$cell ratio.

Conclusion: Short-term omega-3 PUFA administration was associated with reduced postoperative infectious complications, inflammatory cytokines, and hospital stay after CRC surgery. Due to heterogeneity and relatively small sample size, the optimal timing and route of administration deserve further study.

Keywords: omega-3, fatty acids, fish oil, colorectal surgery, meta-analysis

\section{Introduction}

Worldwide, colorectal cancer (CRC) is the third-most common cancer in men, the second-most common cancer in women, and the fourth-most common cause of cancer mortality. ${ }^{1}$ In 2015, it was reported that there were about 1.478 million CRC patients worldwide, which accounted for $9.7 \%$ of total cancer cases, and estimated CRC-caused deaths were $753,000 .^{2}$ Kinds of risk factors and potential factors were found to be relevant to $\mathrm{CRC}$, and subsequently various preventive interventions were investigated., ${ }^{3,4}$ For patients diagnosed with $\mathrm{CRC}$, surgery is still a curative option. However, colorectal surgery was reported to be related to a very high incidence of complication, especially postoperative infections. López et al reported the overall rate of complication was $39.5 \%$, and nearly half of them were infections. ${ }^{5}$ The prevention and treatment of severe postoperative infections of the abdominal and pelvic cavity in CRC patients have always been an important issue for colorectal surgeons.

Omega-3 polyunsaturated fatty acids (PUFAs) are one of the two kinds of essential FAs in humans and must be supplied from outside the body. Two common (c)
hereby accept the Terms. Non-commercial uses of the work are permitted without any further permission from Dove Medical Press Limited, provided the work is properly attributed. For permission hereby accept the Terms. Non-commercial uses of the work are permitted without any further permission from Dove Med
for commercial use of this work, please see paragraphs 4.2 and 5 of our Terms (https://www.dovepress.com/terms.php). 
forms of omega-3 PUFAs are eicosapentaenoic acid and docosahexaenoic acid, both found in fish oil and both with nutritional and pharmacological effects. ${ }^{6}$ Currently, omega-3 PUFA enriched enteral nutrition (EN) and parenteral nutrition $(\mathrm{PN})$ are regarded as one kind of immunonutrition therapy in both intensive care unit and surgical patients. ${ }^{7}$ Although no significant association between omega-3 PUFA supplements and cancer-incidence reduction has been found, ${ }^{8}$ its positive roles on host immune function seem to be promising in the postoperative management of cancer patients. Also, previous meta-analyses including all kinds of surgical patients indicated that omega-3 PUFAs improved clinical outcomes, such as reduced infection incidence and hospital stay. ${ }^{9,10}$

However, due to the influence of different diseases and surgeries, the findings would be difficult to be applied to clinical practice in specific CRC patients. Other studies have investigated the efficacy of omega-3 PUFA-enriched nutrition for CRC patients undergoing surgery, ${ }^{11-21}$ and the primary results indicated that the immunological function of omega-3 PUFAs would be helpful in preventing postoperative infectious complications. Considering the results and conclusions in these studies were not completely consistent because of limited sample size, different study designs, and potential bias, we performed a meta-analysis of all relevant randomized control trials (RCTs) to focus mainly on the efficacy of omega-3 PUFAs in the prevention of postoperative complications for CRC patients undergoing surgery.

\section{Materials and methods}

\section{Literature-search strategy}

We searched the online databases of PubMed (January 1966 to March 2016), the Cochrane Library (2016, issue 3), and Embase (January 1974 to March 2016) by using free terms as follows: (“omega-3" OR “n-3” OR “polyunsaturated”) AND ("fatty acid" OR "fish oil") AND ("cancer" OR "carcinoma” OR “tumor” OR “surgery” OR “operation”) AND (“colorectal” OR “colon" OR "rectum"). Related articles on PubMed and Google Scholar and references of related reviews were also used and screened to find potential literature.

\section{Inclusion process}

Clinical studies investigating the efficacy of short-term omega-3 PUFA-enriched nutrition in CRC patients undergoing surgery were eligible. After duplicates had been removed, the searched citations were firstly screened on the basis of titles and abstracts, and then potential studies were evaluated by reading the full texts to ensure their suitability of inclusion. The study had to be an RCT, and omega-3 PUFAs ideally needed to be administered additionally in the study group (omega-3 group). The daily dose of omega-3 PUFAs was not limited; the route of administration needed to be oral or though enteral tube in EN or intravenous infusion in PN; the timing of administration had to be short-term duration before or after surgery, or both before and after surgery. The inclusion process was completed by two independent reviewers, and only articles published in English were considered.

\section{Outcome measures}

Primary outcome measures needed to include at least the incidence of infectious complications, surgical site infection (SSI), or total complications. Secondary outcome measures would include serum inflammatory cytokines (tumor necrosis factor $[\mathrm{TNF} \alpha]$ and interleukin-6 [IL-6]), $\mathrm{CD} 4^{+}: \mathrm{CD} 8^{+}$cell ratio, hospital stay, and medical cost. As reported, all outcome measures were collected during both hospital stay and follow-up period.

\section{Data extraction and quality assessment}

We extracted both information and outcome data from the included studies. Cases, age, sex, interventions (daily dose, timing, and duration of omega-3 PUFA administration), operation time, and blood loss are presented to show the baseline characteristics. Data of outcomes were extracted as mentioned earlier, including primary and secondary outcome measures. Quality assessment was performed by using the Cochrane bias-risk tool, ${ }^{22}$ which includes six domains: selection bias, performance bias, detection bias, attrition bias, reporting, bias and other bias.

\section{Statistical analysis}

Data synthesis was performed by Reviewer Manager (RevMan 5.3; Cochrane Collaboration, London, UK). Because the timing of preoperative or postoperative administration was obviously of shorter duration than perioperative administration, a subgroup separating administration timing was firstly established to reduce clinical heterogeneity. Then, statistical heterogeneity was calculated by $\chi^{2}$ and $I^{2}$ statistical tests, and a random-effect model or a fixed-effect model was chosen accordingly. Risk ratios (RRs), mean difference (MD), or standard MD (SMD) with 95\% confidence intervals (CIs) were used to show the combined effect size. Sensitivity analyses were performed though changing the synthesis model to test its stability, and inverted funnel plots were visually judged to explore the risk of publication bias. 


\section{Results}

\section{Baseline characteristics and quality of included RCTs}

From 240 identified studies, 223 were excluded on initial screening. After full-text evaluation of the remaining 17 papers, eleven were included (Figure 1). Three papers reported data from the same trial, ${ }^{8-10}$ and one paper reported a trial with three arms, which was regarded as two separate studies, ${ }^{11}$ and thus the combined study contained ten RCTs with a total of $694 \mathrm{CRC}$ patients (348 cases in the omega-3 group and 346 cases in the control group). Detailed baseline characteristics are listed in Table 1. Case numbers ranged from 18 to 148, and average age ranged from 50 to 71 years. Daily administered omega-3 PUFA ranged from 1 to $4 \mathrm{~g}$ in a fixed manner, or 0.05 to $0.2 \mathrm{~g} / \mathrm{kg}$ adjusted to body weight. Preoperative nutrition support was used in three trials, postoperative nutrition support in four trials, and perioperative nutrition support in three trials. Omega-3 PUFA-enriched nutrition was administered orally in five trials: oral + jejunal infusion in one trial and parenteral in four trials.

Overall quality of the RCTs was moderate to high. As shown in Figure 2, four trials had unclear risk in selection bias, and three trials had unclear risk because of a lack of detailed information on random-sequence generation and concealment allocation. ${ }^{11,12,15-17}$ Only one trial showed a high risk of performance and detection bias, as the trial was not blinded. ${ }^{13}$

\section{Effect of omega-3 PUFAs on postoperative infectious complications}

Data for postoperative infectious complications were reported in eight trials. Meta-analyses in a fixed-effect model showed

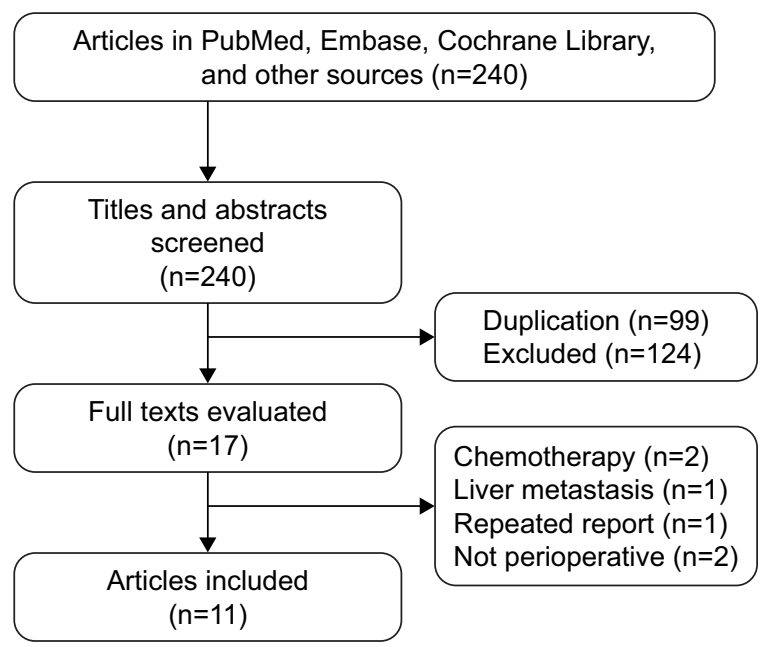

Figure I Flowchart of trial selection.

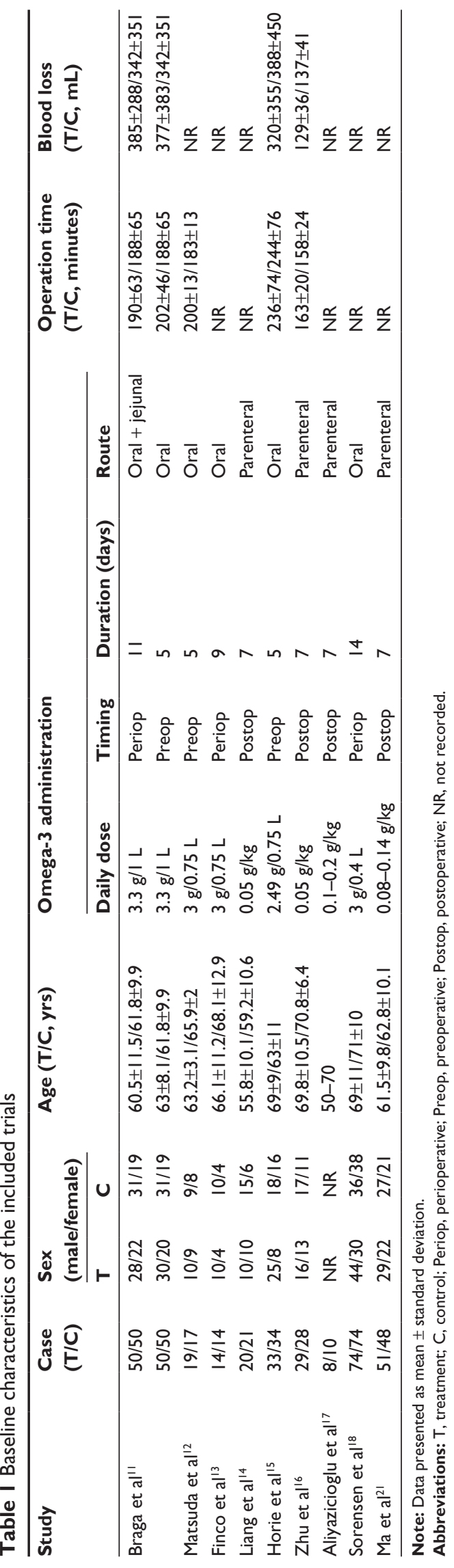




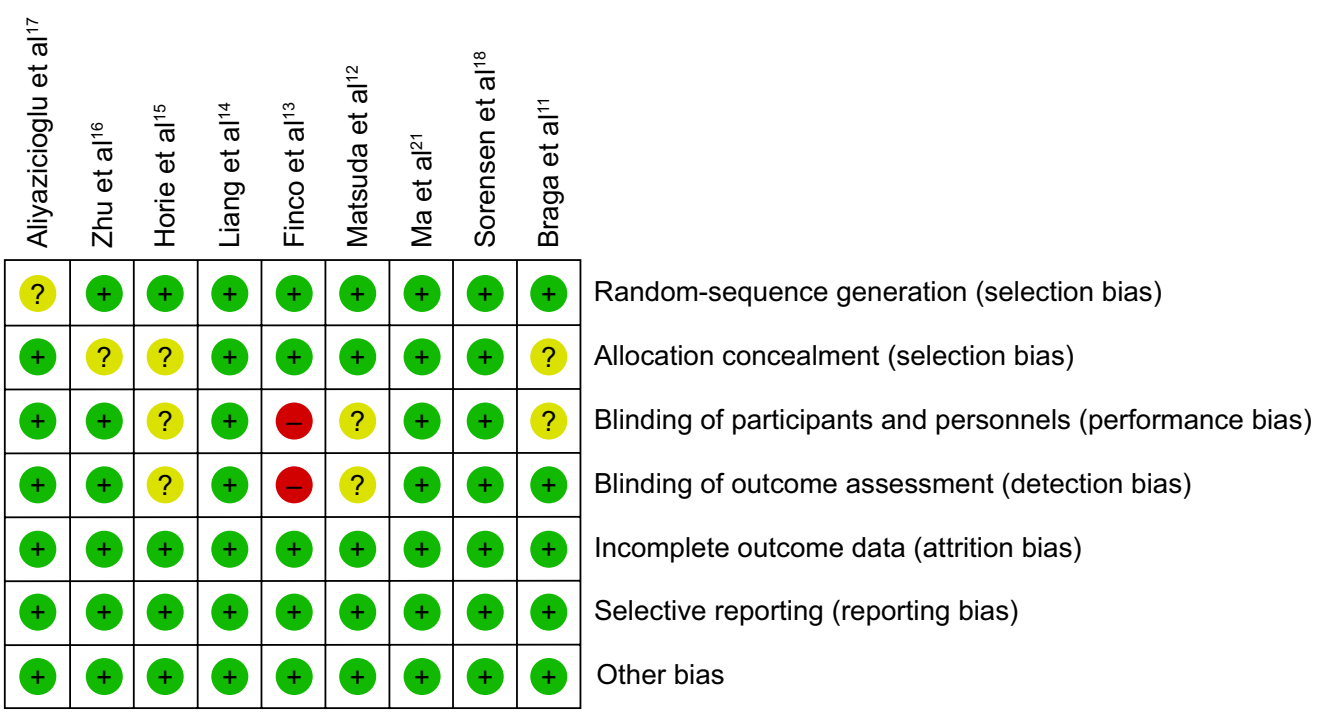

Figure 2 Summary of risk of bias.

that the incidence of postoperative infectious complications was significantly lower in favor of omega-3 PUFAs compared with control ( $15.58 \%$ vs $24.76 \%$, RR $0.63,95 \%$ CI $0.47-0.86$; $P=0.004)$. There was a significant reduction in infectious complications in the pre- and postoperative subgroup ( $7.65 \%$ vs $18.23 \%$, RR $0.43,95 \%$ CI $0.24-0.76 ; P=0.004$ ), though not in the perioperative subgroup (26.09\% vs $33.33 \%$, RR $0.78,95 \%$ CI 0.54-1.13; $P=0.19)$, as shown in Figure 3.

\section{Effect of omega-3 PUFAs on postoperative SSI}

The incidence of SSI was reported in eight trials. Meta-analysis results in a random-effect model revealed no significant difference between omega-3 PUFAs and control (7.17\% vs $10.03 \%$, RR $0.72,95 \%$ CI $0.44-1.2 ; P=0.21$ ). Subgroup analyses likewise showed no significant difference in pre- or postoperative $(4.37 \%$ vs $7.73 \%$, RR $0.58,95 \%$ CI $0.25-1.34$;

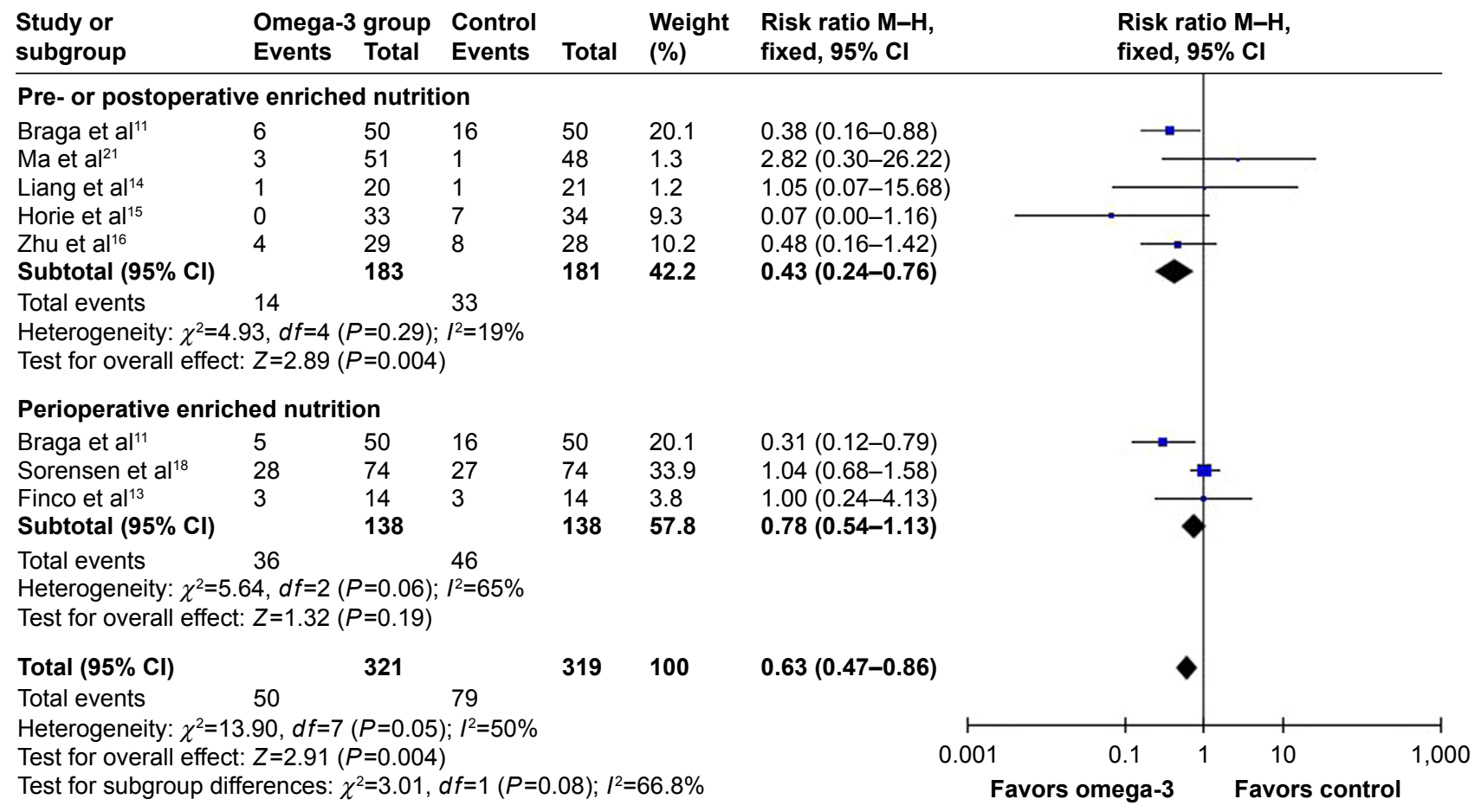

Figure 3 Meta-analysis results of infectious complications among the groups. Abbreviations: $\mathrm{M}-\mathrm{H}$, Mantel-Haenszel; $\mathrm{Cl}$, confidence interval. 
$P=0.2)$ or perioperative $(10.87 \%$ vs $13.04 \%$, RR $0.83,95 \%$

CI $0.44-1.58 ; P=0.58)$ omega-3 PUFA-nutrition support, as shown in Figure 4.

\section{Effect of omega-3 PUFAs on postoperative total complications}

The incidence of postoperative total complications was reported in eight trials. The results revealed no significant difference in either overall meta-analyses (36.76\% vs $47.02 \%$, RR $0.67,95 \%$ CI $0.43-1.04 ; P=0.08$ ) or subgroup analyses of pre- or postoperative ( $36.61 \%$ vs $49.17 \%$, RR $0.53,95 \%$ CI $0.19-1.42 ; P=0.2)$ and perioperative $(36.96 \%$ vs $44.20 \%$, RR 0.82, 95\% CI 0.53-1.25), $P=0.35$ ) omega-3 PUFA administration, as shown in Figure 5.

\section{Effect of omega-3 PUFAs on postoperative inflammatory cytokines and $\mathrm{CD4}^{+}: \mathrm{CD}^{+}$cell ratio}

Inflammatory cytokines were reported in four trials. Metaanalysis results in a fixed-effect model showed that omega-3 PUFA nutrition was associated with a lower level of TNF $\alpha$ (SMD $-0.37,95 \% \mathrm{CI}-0.66$ to $-0.07 ; P=0.01$ ) and IL-6 (SMD $-0.36,95 \% \mathrm{CI}-0.66$ to $-0.07 ; P=0.02$ ) compared with control, with no significant influence on $\mathrm{CD}^{+}: \mathrm{CD}^{+}$ cell ratio (SMD $0.36,95 \% \mathrm{CI}-0.04$ to $0.76 ; P=0.08$ ); as shown in Figure 6.

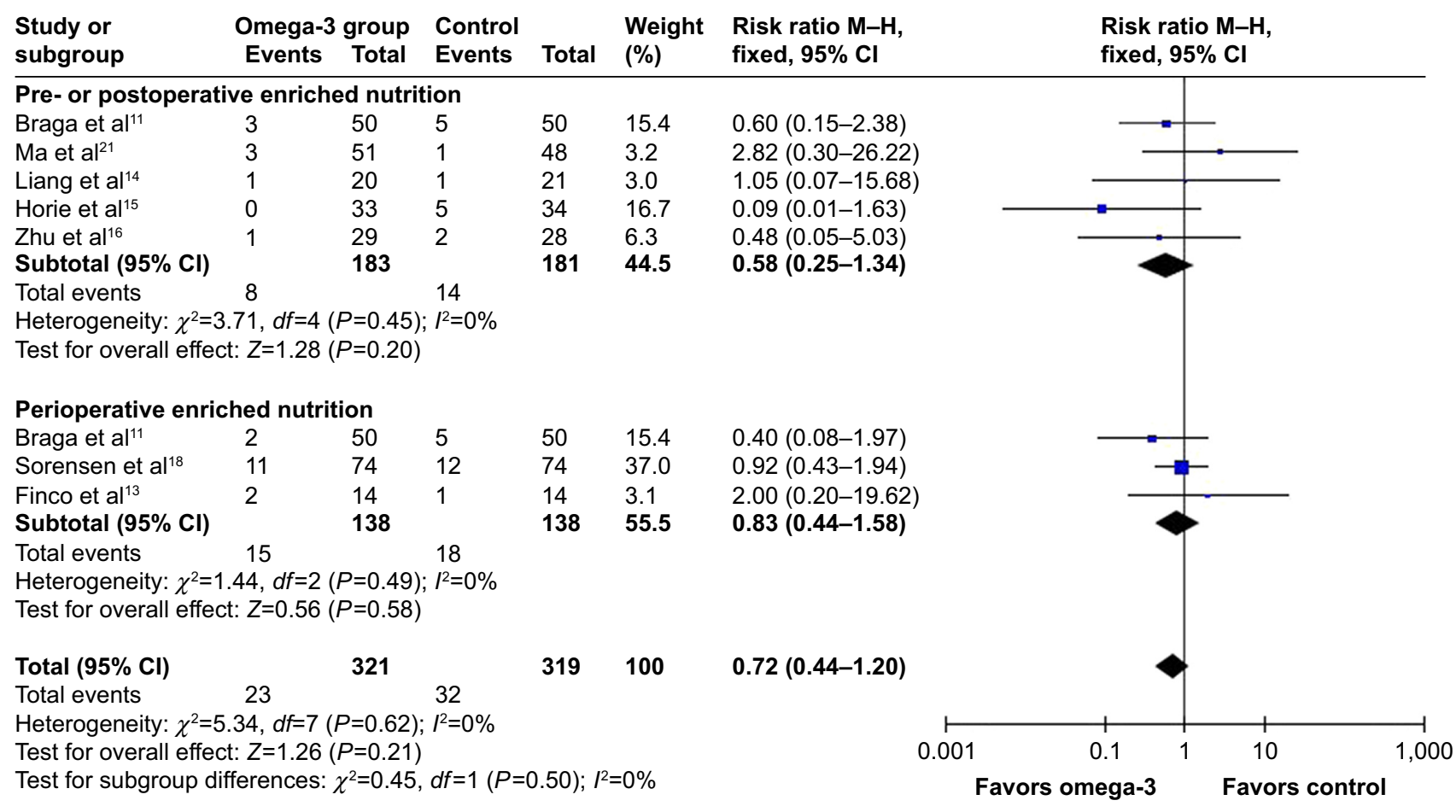

\section{Effect of omega-3 PUFAs on postoperative hospital stay}

Hospital stay was reported in six trials, and meta-analyses in a random-effect model showed a significantly reduced postoperative hospital stay in the omega-3 group compared with the control group (MD $-2.09,95 \%$ CI -3.71 to -0.48 ; $P=0.01$ ), as shown in Figure 7 .

\section{Medical cost analysis}

Only one trial reported nutrition therapy cost and total cost, ${ }^{16}$ and there was no significant difference between the groups in total cost ( $\mathrm{CN} ¥ 38,025 \pm 389.6$ vs $37,968 \pm 563.5, P>0.05)$, although the omega-3 group had significantly higher nutrition therapy cost $(4,025 \pm 309.6$ vs $2,568 \pm 445.2, P<0.01)$.

\section{Sensitivity analysis}

Through changing the synthesis model, sensitivity-analysis results demonstrated that the trends of pre- or postoperative infectious complications, TNF $\alpha$, and hospital stay did not alter, while the trends of overall infectious complications (RR 0.6, 95\% CI 0.34-1.06; $P=0.51$ ) and IL-6 (SMD -0.4, $95 \% \mathrm{CI}-0.82$ to $0.02 ; P=0.06)$ changed.

\section{Publication bias}

Publication bias may have existed in postoperative total complications and hospital stay, while there were low risks

Figure 4 Meta-analysis results of surgical site infections among the groups. Abbreviations: $\mathrm{M}-\mathrm{H}$, Mantel-Haenszel; $\mathrm{Cl}$, confidence interval. 


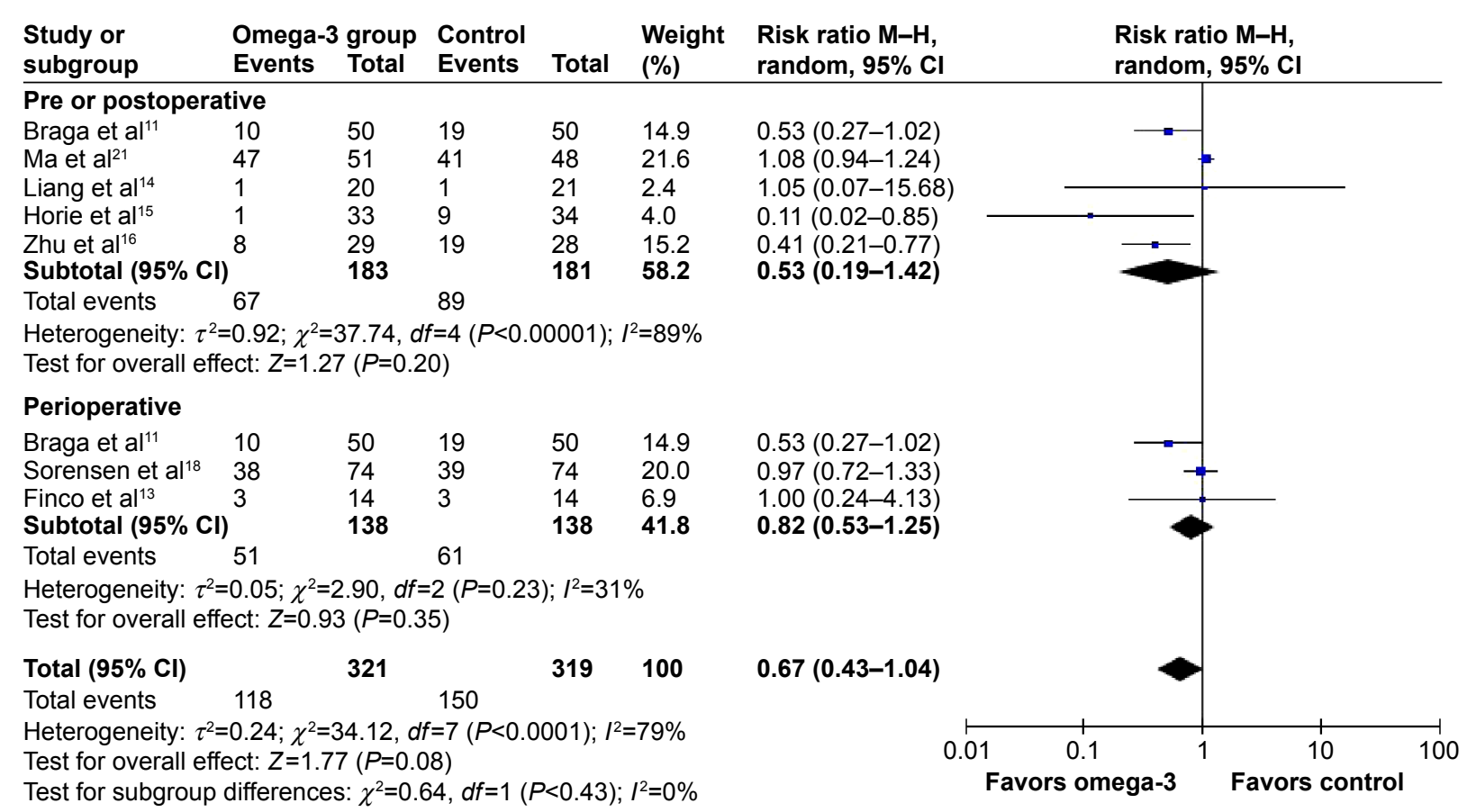

Figure 5 Meta-analysis results of total complications among the groups.

Abbreviations: $\mathrm{M}-\mathrm{H}$, Mantel-Haenszel; $\mathrm{Cl}$, confidence interval.

of publication bias in outcomes of postoperative infectious complications and SSI.

\section{Discussion}

CRC surgery is associated with a high incidence of postoperative infections. The infections always led to prolonged hospital stay, increased medical cost, and even treatment failure. Underlying mechanisms included preoperative intestinal cleansing unsatisfied with residual feces in the colon, intraoperative incision of the colon and postoperative anastomotic leakage, which would highly increase the risk of bacterial contamination to the peritoneal cavity and

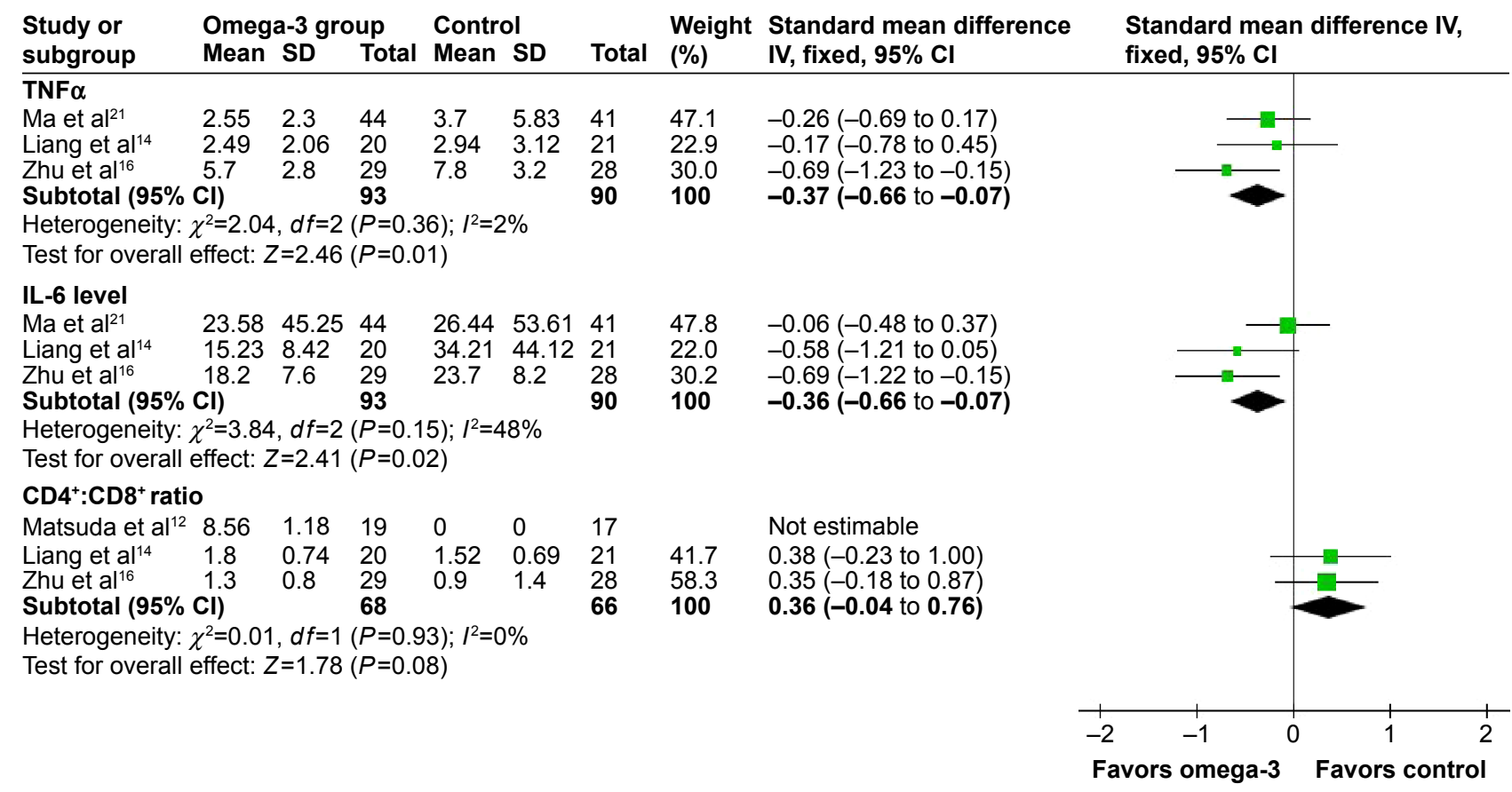

Figure 6 Meta-analysis results of cytokines and $C D 4^{+}: \mathrm{CD}^{+}$cell ratio among the groups.

Abbreviations: SD, standard deviation; IV, instrumental variable; $\mathrm{Cl}$, confidence interval; TNF $\alpha$, tumor necrosis factor; IL-6, interleukin-6. 


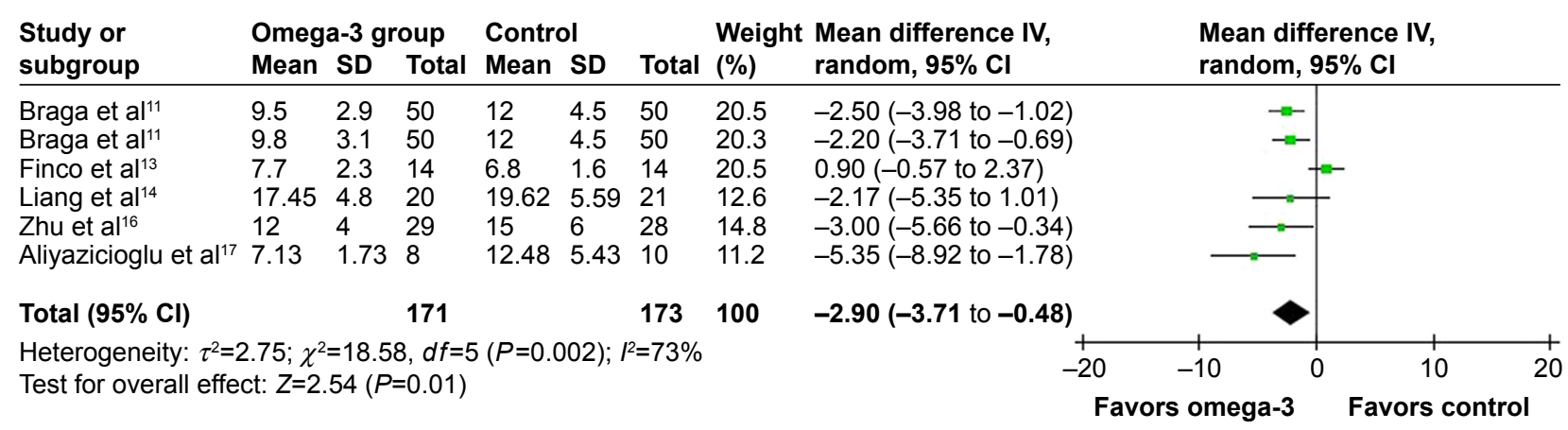

Figure 7 Meta-analysis of hospital stay among the groups.

Abbreviations: SD, standard deviation; IV, instrumental variable; $\mathrm{Cl}$, confidence interval.

surgical wound, together with the suppressed host immune function due to cancer and surgery stress. Among them, impaired immune status in CRC patients and acute stress of CRC surgery were considered to be the most important factors. ${ }^{12}$ In animal and observational clinical studies, omega-3 PUFA-enriched immuonutrition was reported to have antiplatelet, anti-inflammatory, antiangiogenic, and anti-CRC functions. . $^{7,23,24}$

To the best of our knowledge, the current study is the first meta-analysis focused on the clinical efficacy of short-term omega-3 PUFA immuonutrition in the prevention of specific CRC patients at very high risk of infection. Our study demonstrated significant benefits for infectious complication prevention, as well as serum inflammatory cytokines and hospital stay improvements, while no significant influence was found for total complications, SSI, or total medical cost.

Meta-analysis results in a fixed-effect model showed that the omega-3 group had a lower rate of postoperative infectious complications compared with the control group (15.6\% vs $24.8 \%$ ), and the reported infectious complications included the respiratory tract, urinary tract, abdominal abscess, bacteremia, and wound infection. Further subgroup analyses according to timing of omega-3 PUFA administration revealed that this significant difference would have been mainly contributed to by the pre- or postoperative subgroup. Perioperative omega-3 PUFA-enriched nutrition was administered for 9-14 days, while pre- or postoperative omega-3 PUFAs were always administered for 5-7 days. As such, the perioperative subgroup had an obviously longer duration of administration than pre- and postoperative subgroup, while no significant difference was found in the perioperative group. With regard to the optimal timing of immuonutrition administration, some studies stated preoperative nutrition may be helpful for the body to obtain an adequate level in time for the stress of surgery, and early postoperative nutrition was important issue fast recovery of intestinal function and psychological status. ${ }^{13,25}$ A recent network meta-analysis indicated that perioperative enteral immuonutrition (EIN) was better than pre- and postoperative EIN for postoperative infectious complication prevention. ${ }^{26}$ The network analysis also revealed that the timing of nutrition support seemed to have different influences on different outcome measures.

Possible explanations of the negative result of perioperative omega-3 PUFA administration in the current study were as follows. First, the sample size in the perioperative subgroup may be under the test power. It has been reported that 148 cases were enough to detect a $20 \%$ reduction in the rate of infectious complications, ${ }^{19}$ whereas an expected reduction in our study was only $9.2 \%$, and thus nearly 300 cases were required (when $\beta=0.8, \alpha=0.05$ ). Second, the perioperative subgroup included only patients receiving EIN orally or through an enteral tube, while the postoperative group included patients receiving PN through intravenous infusion. For postoperative nutrition administration, EN had advantages in aspects of commensal bacteria balance and intestinal function recovery, and was also a key intervention in the principle of fast-track surgery. ${ }^{13,27}$ Gastric paralysis lasted for 24 hours, colon dysfunction lasted for about 48 hours after abdominal surgery, ${ }^{13,28}$ and the time would be even longer in elective CRC surgery. Therefore, omega-3 PUFA-enriched EIN in the perioperative group during the early postoperative period may have been badly absorbed and utilized. Third, the different dose and formulation of omega-3 PUFAs in the studies might also have had an influence to the outcomes.

The current study also investigated changes in inflammatory cytokines, and the results showed that serum levels of TNF $\alpha$ and IL- 6 were lower, demonstrating the anti-inflammatory role of omega-3 PUFAs. Both of the two cytokines would induce $\mathrm{CD} 4^{+} \mathrm{T}$ cells differentiated from different T-helper $\left(\mathrm{T}_{\mathrm{h}}\right)$ cells, and the levels of TNF $\alpha / \mathrm{IL}-6$ to some extent reflected the situation of $\mathrm{T}_{\mathrm{h}} 1-\mathrm{T}_{\mathrm{h}} 2$ cell balance. 
$\mathrm{T}_{\mathrm{h}} 1$ cells mainly mediate cellular immune response and play important roles in infection and tumor defense, while $\mathrm{T}_{\mathrm{h}} 2$ cells mainly mediate humoral immune response. CRC patients always had a $T_{h} 1-T_{h} 2$ imbalance shifted to $T_{h} 2$, and together with surgery stress this imbalance was enhanced and reported to be highly associated with postoperative infections. ${ }^{29,30}$ However, sensitivity analysis in our study suggested that IL-6 changes were not stable, so whether omega-3 PUFA-enriched nutrition modulated the $T_{h} 1-T_{h} 2$ balance or not was unclear. Due to the limited studies, its effects on $\mathrm{CD}^{+}$and $\mathrm{CD}^{+} \mathrm{T}$-cell balance was also unclear.

\section{Limitations}

There were several limitations in our study. Although qualityassessment results indicated the overall study quality was good, several trials had some risk of bias in their study design, as mentioned earlier. Publication bias always exists and is unavoidable in meta-analyses, and inverted funnel plots of our study indicated potential risks in outcomes of total complication and hospital stay. For infectious outcomes, different doses and kinds of antibiotic used before and after surgery may have caused heterogeneity across the trials. Although the preoperative prophylactic antibiotic was comparable in each separate trial, the intra- and postoperative antibiotic can only be used based on a certain situation of operation time and blood loss, while only half of the trials reported relevant data. SSI was also an important issue in the clinic, and CRC surgery as a clean-contaminated operation had a high risk of SSI, ${ }^{31}$ although the omega-3 group had a lower SSI incidence and no statistical difference was found. As Braga et al reported, about $25 \%$ of postoperative infection, especially wound infection, occurred after discharge, ${ }^{11}$ and longer follow-up to 1 month would be important for future study.

\section{Conclusion}

Short-term omega-3 PUFAs were associated with reduced postoperative infectious complications, inflammatory cytokines, and hospital stay after CRC surgery. Due to heterogeneity and relatively small sample size, the optimal timing and route of administration deserve further longer follow-up study.

\section{Disclosure}

The authors report no conflicts of interest in this work.

\section{References}

1. Aran V, Victorino AP, Thuler LC, Ferreira CG. Colorectal cancer: epidemiology, disease mechanisms and interventions to reduce onset and mortality. Clin Colorectal Cancer. 2016;15(3):195-203.
2. Ferlay J, Soerjomataram I, Dikshit R, et al. Cancer incidence and mortality worldwide: sources, methods and major patterns in GLOBOCAN 2012. Int J Cancer. 2015;136(5):E359-E386.

3. Gagnière J, Raisch J, Veziant J, et al. Gut microbiota imbalance and colorectal cancer. World J Gastroenterol. 2016;22(2):501-518.

4. Sugarbaker PH. Colorectal cancer: prevention and management of metastatic disease. Biomed Res Int. 2014;2014:782890.

5. López PR, Escribano JA, Rodríguez-Cuéllar E, García IL, Mata EJ. Proyecto nacional para la gestión clínica de procesos asistenciales. Tratamiento quirúrgico del cáncer colorrectal. I. Aspectos generales [National project for the management of clinical processes: surgical treatment of colorectal cancer - I: general aspects]. Cir Esp. 2002;71(4):173-180. Spanish.

6. Hull MA. Omega-3 polyunsaturated fatty acids. Best Pract Res Clin Gastroenterol. 2011;25(4):547-554.

7. Pradelli L, Mayer K, Muscaritoli M, Heller AR. n-3 Fatty acid-enriched parenteral nutrition regimens in elective surgical and ICU patients: a meta-analysis. Crit Care. 2013;16(5):R184.

8. Zhang YF, Gao HF, Hou AJ, Zhou YH. Effect of omega-3 fatty acid supplementation on cancer incidence, non-vascular death, and total mortality: a meta-analysis of randomized controlled trials. BMC Public Health. 2014;14:204.

9. Wei C, Hua J, Bin C, Klassen K. Impact of lipid emulsion containing fish oil on outcomes of surgical patients: systematic review of randomized controlled trials from Europe and Asia. Nutrition. 2010;26(5): 474-481.

10. Chen B, Zhou Y, Yang P, Wan HW, Wu XT. Safety and efficacy of fish oil-enriched parenteral nutrition regimen on postoperative patients undergoing major abdominal surgery: a meta-analysis of randomized controlled trials. JPEN J Parenter Enteral Nutr. 2010;34(4): 387-394.

11. Braga M, Gianotti L, Vignali A, Carlo VD. Preoperative oral arginine and n-3 fatty acid supplementation improves the immunometabolic host response and outcome after colorectal resection for cancer. Surgery. 2002;132(5):805-814.

12. Matsuda A, Furukawa K, Takasaki H, et al. Preoperative oral immuneenhancing nutritional supplementation corrects $\mathrm{TH} 1 / \mathrm{TH} 2$ imbalance in patients undergoing elective surgery for colorectal cancer. Dis Colon Rectum. 2006;49(4):507-516.

13. Finco C, Magnanini P, Sarzo G, et al. Prospective randomized study on perioperative enteral immunonutrition in laparoscopic colorectal surgery. Surg Endosc. 2007;21(7):1175-1179.

14. Liang B, Wang S, Ye YJ, et al. Impact of postoperative omega-3 fatty acid-supplemented parenteral nutrition on clinical outcomes and immunomodulations in colorectal cancer patients. World J Gastroenterol. 2008; 14(15):2434-2439.

15. Horie H, Okada M, Kojima M, Nagai H. Favorable effects of preoperative enteral immunonutrition on a surgical site infection in patients with colorectal cancer without malnutrition. Surg Today. 2006;36(12): 1063-1068.

16. Zhu MW, Tang DN, Hou J, et al. Impact of fish oil enriched total parenteral nutrition on elderly patients after colorectal cancer surgery. Chin Med J (Engl). 2012;125(2):178-181.

17. Aliyazicioglu T, Cantürk NZ, Şimşek T, Kolayli F, Çekmen M. Effects of standard and/or glutamine dipeptide and/or omega-3 fatty acidsupplemented parenteral nutrition on neutrophil functions, interleukin-8 level and length of stay-a double blind, controlled, randomised study. East Afr Med J. 2014;90(2):59-66.

18. Sorensen LS, Rasmussen HH, Aardestrup IV, et al. Rapid incorporation of $\omega-3$ fatty acids into colonic tissue after oral supplementation in patients with colorectal cancer: a randomized, placebo-controlled intervention trial. JPEN J Parenter Enteral Nutr. 2014;38(5):617-624.

19. Sorensen LS, Thorlacius-Ussing O, Rasmussen HH, et al. Effects of perioperative supplementation with omega-3 fatty acids on leukotriene B4 and leukotriene B5 production by stimulated neutrophils in patients with colorectal cancer: a randomized, placebo-controlled intervention trial. Nutrients. 2014;6(10):4043-4057. 
20. Sorensen LS, Thorlacius-Ussing O, Schmidt EB, et al. Randomized clinical trial of perioperative omega-3 fatty acid supplements in elective colorectal cancer surgery. Br J Surg. 2014;101(2):33-42.

21. Ma CJ, Wu JM, Tsai HL, et al. Prospective double-blind randomized study on the efficacy and safety of an n-3 fatty acid enriched intravenous fat emulsion in postsurgical gastric and colorectal cancer patients. Nutr J 2015;14:9.

22. Higgins JP, Altman DG, Gøtzsche PC, et al. The Cochrane Collaboration's tool for assessing risk of bias in randomised trials. BMJ. 2011; 343:d5928.

23. Roulet M, Frascarolo P, Pilet M, Chapuis G. Effects of intravenously infused fish oil on platelet fatty acid phospholipid composition and on platelet function in postoperative trauma. JPEN J Parenter Enteral Nutr. 1997;21(5):296-301.

24. Cockbain AJ, Toogood GJ, Hull MA. Omega-3 polyunsaturated fatty acids for the treatment and prevention of colorectal cancer. Gut. 2014; 61(4):135-149.

25. Gianotti L, Braga M, Nespoli L, Radaelli G, Beneduce A, Di Carlo V. A randomized controlled trial of preoperative oral supplementation with a specialized diet in patients with gastrointestinal cancer. Gastroenterology. 2002;122(7):1763-1770.
26. Song GM, Tian X, Zhang L, et al. Immunonutrition support for patients undergoing surgery for gastrointestinal malignancy: preoperative, postoperative, or perioperative? A Bayesian network meta-analysis of randomized controlled trials. Medicine (Baltimore). 2015;94(29):e1225.

27. Desai SV, Mcclave SA, Rice TW. Nutrition in the ICU: an evidencebased approach. Chest. 2014;145(5):1148-1157.

28. Bufo AJ, Feldman S, Daniels GA, Lieberman RC. Early postoperative feeding. Dis Colon Rectum. 1994;37(12):5-6.

29. Tabata T, Hazama S, Yoshino S, Oka M. Th2 subset dominance among peripheral blood $\mathrm{T}$ lymphocytes in patients with digestive cancers. Am J Surg. 1999;177(3):203-208.

30. Tatsumi $\mathrm{H}, \mathrm{Ura} H$, Ikeda $\mathrm{S}$, et al. Surgical influence on $\mathrm{TH} 1 / \mathrm{TH} 2$ balance and monocyte surface antigen expression and its relation to infectious complications. World J Surg. 2003;27(5):522-528.

31. National Nosocomial Infections Surveillance System. National Nosocomial Infections Surveillance (NNIS) System Report, data summary from January 1992 through June 2004, issued October 2004. Am J Infect Control. 2004;32(8):470-485.
OncoTargets and Therapy

\section{Publish your work in this journal}

OncoTargets and Therapy is an international, peer-reviewed, open access journal focusing on the pathological basis of all cancers, potential targets for therapy and treatment protocols employed to improve the management of cancer patients. The journal also focuses on the impact of management programs and new therapeutic agents and protocols on

\section{Dovepress}

patient perspectives such as quality of life, adherence and satisfaction. The manuscript management system is completely online and includes a very quick and fair peer-review system, which is all easy to use. Visit http://www.dovepress.com/testimonials.php to read real quotes from published authors. 